\title{
Delayed dispersal as a potential route to cooperative breeding in ambrosia beetles
}

\author{
Katharina Peer • Michael Taborsky
}

Received: 9 January 2006 /Revised: 3 August 2006 / Accepted: 29 October 2006 / Published online: 19 December 2006

(C) Springer-Verlag 2006

\begin{abstract}
Xyleborini are a species-rich tribe of ambrosia beetles, which are haplodiploid and typically mate among siblings within their natal brood chamber. Several characteristics of this tribe would predict the evolution of higher levels of sociality: high genetic relatedness within galleries due to inbreeding, high costs of dispersal and the potential benefit of cooperation in brood care within the natal gallery (e.g. by fungus gardening, gallery extension, offspring feeding and cleaning). However, information on the social system of these beetles is very limited. We examined the potential for cooperative breeding in Xyleborinus saxeseni by monitoring dispersal in relation to brood size and composition. Results show that adult female offspring delay dispersal despite dispersal opportunities, and apparently some females never disperse. The females' decision to stay seems to depend on the presence of eggs and dependent siblings. We found no indication that female offspring reproduce in their natal gallery, as colonies with many mature daughters do not contain more eggs than those with few or no daughters. There is a significant positive relationship between the number of females present and the number of dependent siblings (but not eggs), which suggests that cooperative brood care of female offspring raises colony productivity by improving survival rates of immatures. Our results suggest that cooperative breeding is
\end{abstract}

Communicated by D. Gwynne

K. Peer $\cdot$ M. Taborsky $(\bowtie)$

Department of Behavioural Ecology, Institute of Zoology,

University of Berne,

Wohlenstr. 50a,

CH-3032 Hinterkappelen, Switzerland

e-mail: michael.taborsky@esh.unibe.ch likely to occur in $X$. saxesen $i$ and possibly other xyleborine species. We argue that a closer look at sociality within this tribe may yield important information on the factors determining the evolution of cooperative breeding and advanced social organization.

Keywords Cooperative agriculture $\cdot$ Kin selection . Fungus cultivation $\cdot$ Haplodiploidy $\cdot$ Inbreeding . Ecological constraints $\cdot$ Reproductive skew

\section{Introduction}

Advanced social organization and cooperative breeding have been described for a wide range of animal taxa. Eusociality involving complete reproductive skew between classes of individuals (a single reproductive individual and a number of non-reproductive helpers) can be regarded as one extreme in a continuum of cooperative breeding in animals (Sherman et al. 1995) - the other end of the continuum being the equal share of reproduction between individuals of a society. For decades, research on social evolution in invertebrates and vertebrates has addressed different questions. Studies on vertebrate social systems mainly investigated ecological factors responsible for cooperative breeding, whereas for invertebrates, the original focus was on kin selection benefits and relatedness asymmetries due to haplodiploidy - mainly in the context of eusociality. However, eusociality has been discovered in a variety of widely distinct taxa (see Aoki 1977; Alexander et al. 1991; Kent and Simpson 1992; Crespi 1992; Duffy 1996; Thorne 1997), many of which are not haplodiploid. Therefore, there is increasing interest to develop a general framework of social evolution that includes both invertebrates and vertebrates (Roisin 1999). Most research on the 
evolution of social systems has focused on variation of phenotypes within social species. However, to truly understand the selective forces involved at the origin of sociality, comparative studies on social and non-social sister taxa are essential (see Crespi and Choe 1997). In this study, we examine several life-history characteristics in a species of Xyleborini (Scolytidae), an entirely haplodiploid tribe of fungus-cultivating beetles ("ambrosia beetles"). A closer investigation of this tribe, which may display both social and non-social sister taxa, will be particularly useful for understanding the relative importance of ecological and life-history characteristics involved in the transition from solitary to cooperative breeding and advanced sociality.

Insect fungus cultivation requires continuous maintenance, which may be accomplished more easily by the cooperation of labourers instead of the sole effort of a single mother. The great potential of cooperative agriculture is reflected by the enormous productivity of leaf-cutter ants in the neotropics and macrotermitine termites in the palaeotropics (Bignell and Eggleton 2000; Aanen et al. 2002). Whereas all species in these taxa are eusocial (Aanen et al. 2002), eusociality has been suggested only for one species of ambrosia beetle up to now (Kent and Simpson 1992) and not for any member of the Xyleborini. However, these beetles also cultivate fungus, and all ambrosia beetles show intensive parental care by one or both parents (Kirkendall et al. 1997). Participation by adult offspring in raising siblings could increase brood care efficiency in this group due to the maintenance requirements of fungi without using up the required substrate, as large dead trees are an abundant resource for these small beetles once colonized (size range of one to a few millimetres).

Within the ambrosia beetles, the tribe Xyleborini is the largest, including more than 1,200 species (Normark et al. 1999). Their rapid diversification may have resulted from the combined action of inbreeding and fungus cultivation (Jordal et al. 2000). The use of fungi makes it possible to use a great variety of plant taxa, and inbreeding confers a colonization advantage and promotes reproductive isolation. The entire tribe exhibits haplodiploid sex determination and is characterized by high levels of inbreeding (Kirkendall 1993; Normark et al. 1999; Peer and Taborsky 2005). Sex ratios are highly female-biased, and sexual dimorphism is pronounced, with males generally being smaller than females, having reduced eyes and nonfunctional flight wings. Mating takes place almost exclusively between siblings within the natal brood chamber (but see Peer and Taborsky 2004). If the negative effects of competition between kin do not increase at a similar or even higher rate as the positive effects of cooperation (West et al. 2002), the overall high relatedness in these beetles could predispose the whole tribe to the evolution of cooperative breeding.

Instead of classifying social groups into descriptive categories, it may be more useful to examine an individual's decisions and their ecological correlates within a "social trajectory" (Helms Cahan et al. 2002). Constraints on independent reproduction and benefits of philopatry can be seen as two sides of the same coin (Koenig et al. 1992; Emlen 1994). Three key decisions are involved in becoming social: whether or not to disperse, whether or not to breed in the natal group and whether or not to cooperate in raising offspring. At each decision point, individuals should be selected to weigh the costs and benefits of alternative strategies.

If the probability of independent reproduction is relatively low, on average, it may be more profitable to remain in the natal gallery and to help in raising siblings until resource deterioration sets an end to brood production. As ambrosia beetles usually depend on the presence of freshly dead trees for gallery initiation, successful host location is unpredictable. In addition, their small size makes them extremely vulnerable to predation or adverse environmental conditions like strong winds during dispersal. Once a suitable host tree has been detected, a foundress must excavate a gallery and overcome remaining tree defence mechanisms like resins. Then she must successfully establish the ambrosia fungus within the gallery before she can start oviposition. The whole process is time-consuming and risky. Multiple founding of galleries has never been observed in this species. Helping in an already established, productive gallery is likely to be an advantageous alternative to individual gallery foundation (see Gadagkar 1990). Therefore, higher levels of sociality should be expected in many xyleborine species, depending on characteristics of their ecology and intrinsic life-history traits.

The aim of this study was to examine life-history traits in Xyleborinus saxeseni, a likely candidate for cooperative breeding because of its large brood sizes and long-lasting colonies. Specifically, we tested three predictions concerning the three main decisions about dispersal, breeding and helping:

(1) Do females delay dispersal? If they do so, adult female offspring should be present in galleries despite existing dispersal opportunities.

(2) Do females reproduce in their natal colony? If females refrain from reproduction in natal galleries, the number of eggs should not increase with the number of adult female offspring in the gallery.

(3) Do females help to raise their dependent siblings in the natal galleries? If females show alloparental care, the presence of adult female offspring should increase colony productivity. 


\section{Materials and methods}

\section{Study system}

The study was conducted in forests surrounding Berne, Switzerland, from April 2001 until June 2002. X. saxeseni is one of seven xyleborine ambrosia beetle species in Europe (Schwenke 1974). After dispersal flight, each founding female initiates a gallery in a freshly dead treepreferably in crevices in the bark - and cultivates ambrosia fungus (Ambrosiella sulfurea) on the surface of the gallery walls. After the fungus has started to grow, she starts oviposition. In contrast to many other xyleborine species, the eggs are not laid in separate branches of the main tunnel, but all offspring develop together in one central brood chamber in close contact with each other. All offspring feed on the ambrosia fungus (Fischer 1954). Brood sizes are large, reaching several hundred in some cases, and overwintering may take place at all stages from egg to adult (Fischer 1954). Earlier anecdotal observations suggest that the foundress excavates a small initial chamber, which is then extended by adult female offspring and perhaps also by larvae (Eichhoff 1881; Hopkins 1898). This has been confirmed by preliminary lab observations of specimens bred in artificial medium (P. Biedermann, personal communication). No morphological differences between foundress and daughters could be detected (unpublished data). It is unlikely that males play a significant role in gallery maintenance because of the strong sexual dimorphism and the small number of males (sex ratios on average amount to 1:20; unpublished data). Dispersing females are already fertilized and found new colonies, whereas males die in their natal gallery after mating (Hopkins 1898; Hosking 1972).

\section{Dispersal monitoring}

The pattern of dispersal activity in a species provides information about dispersal options of individuals throughout the year. The timing of dispersal in $X$. saxesen $i$ and the number of generations within one season have been debated in the literature (Eichhoff 1881; Fischer 1954): whereas some authors have observed two peaks in the dispersal flight and therefore concluded that there is probably more than one generation per season, others found no new colonization events after spring dispersal, thereby excluding a second generation. The occurrence of dispersal throughout the season is an important prerequisite for offspring options of dispersal vs cooperative brood care. If gallery founding is impossible later in the season, daughters would have no choice but to wait in their natal gallery until the next spring.

We monitored the dispersal flight of $X$. saxeseni by setting up 11 ethanol-baited traps in three different forests.
Dispersing females use ethanol vapour from decaying wood to locate suitable host trees. Individuals captured in the traps can thus be assumed to be dispersing individuals attempting to establish their own gallery. The traps were checked twice a week throughout the season, i.e. from April to October. In addition, dispersal from individual galleries was monitored using "emergence" traps. Small plastic vials with a hole in the bottom were attached to the entrance holes of galleries (for details, see Peer and Taborsky 2004). Dispersing individuals were trapped in these vials, and the traps were also checked twice a week. The number of individuals was divided by the number of days since the last check to obtain mean daily dispersal rates.

\section{Gallery surveillance}

To evaluate gallery success, opportunity for cooperative brood care (overlap of generations) and potential benefits of helping behaviour (increased productivity), brood size and composition (age classes) were recorded at different stages of gallery development.

Throughout the study period, we marked and numbered gallery entrances in standing dead trees. Each gallery was checked every 3 to 8 days throughout the study period either until dissection (see below) or until gallery failure was determined. At each check, the presence of a female blocking the gallery entrance (usual behaviour for all bark and ambrosia beetles) and the presence and amount of fresh sawdust and debris in and around the entrance hole were recorded. If there was no sign of activity for several weeks and/or the entrance tunnel was overgrown with fungus, a gallery was classified as failed. Failure was confirmed by dissection of a sample of those galleries (as described below). At galleries with emergence traps, we collected ejected frass and recorded its amount and composition (fresh sawdust, faeces, fungal debris and sometimes dead body parts) to obtain a rough estimate of the size and stage of these colonies. Galleries were carved out and dissected at regular intervals (on average, ten galleries per month) so gallery composition (number of individuals and their age stages) could be determined at different stages and at different times of the year. For sampling, small blocks of wood containing the gallery were removed using hammer and chisel.

In the lab, these blocks were carefully split apart, and gallery composition was recorded. We distinguished between three different larval stages based on estimates of the head capsule diameter (Fischer 1954; Hosking 1972). The age of adult individuals can be roughly estimated based on coloration. Freshly eclosed individuals are very pale, attaining their black coloration after several days to a few weeks; thus, we distinguished between young (pale) and old (black) adult females. Because of the small size and 
delicate nature of the beetles, they were often damaged or killed during sampling. We found that it was physically almost impossible to extract a block of wood with a diameter of a few centimetres and a depth of up to $10 \mathrm{~cm}$ from a log without damage. Thus, in many cases, it could not be determined with certainty whether individuals found dead in the gallery had died already before the extraction. However, in some galleries, undamaged but decaying beetles were found packed in debris and confined to certain areas of the gallery (described as "death chambers" by Hopkins 1898). In these galleries, the presence and number of individuals that had died before excavation and without the observer's influence could be unequivocally determined. From galleries equipped with an emergence trap, we also obtained data on dispersing individuals.

\section{Sample sizes}

Gallery composition could only be determined at a single point in time for each gallery because of the destructive nature of the sampling process (gallery dissection). As data selection for various analyses was often dependent on gallery composition, which was unknown before sampling, sample sizes differed between individual analyses (Fig. 1). In total, 407 galleries on ten different trees were marked and monitored. Of these, 102 galleries were dissected. Ninety-two $(90 \%)$ of the dissected galleries originated from five trees; the remaining $10 \%$ were distributed over five further trees. For 39 galleries, we recorded the distance between the gallery entrance and the nearest neighbour gallery as a measure of density. For analyses concerning the potential helping behaviour (opportunities for cooperative brood care, gallery productivity and multiple egg laying), we included only galleries that had produced at least one offspring until the time of dissection $(n=72)$.

Fifty-eight of the galleries were equipped with emergence traps before dissection, thus yielding information about dispersal. For analyses about dispersal patterns and cues, only galleries where dispersal could potentially occur (i.e. where adult female offspring were present or had already dispersed at the time of dissection) were used $(n=$ 28). The relatively small sample of galleries where adult female offspring were present before dispersal had started $(n=8)$ is due to the limited information about gallery composition before sampling.

\section{Statistical analyses}

For certain analyses (as indicated by the terms used in the "Results"), different age cohorts were grouped to reduce the number of variables contained in the models: all larval stages were pooled to obtain the total number of larvae; larvae and pupae were pooled to represent the number of dependent siblings/brood; and the total number of adult females was calculated including both young and old females.

The raw data on counts of individuals at different stages were not normally distributed (one-sample KolmogorovSmirnov test). Therefore, we used non-parametric statistics
Fig. 1 Sampling scheme and sample sizes of galleries used in the analyses. The boxes represent subsamples of all marked galleries. The two subsamples at each level are part of the left subsample at the level above. Only subsamples in bold were used in the analyses

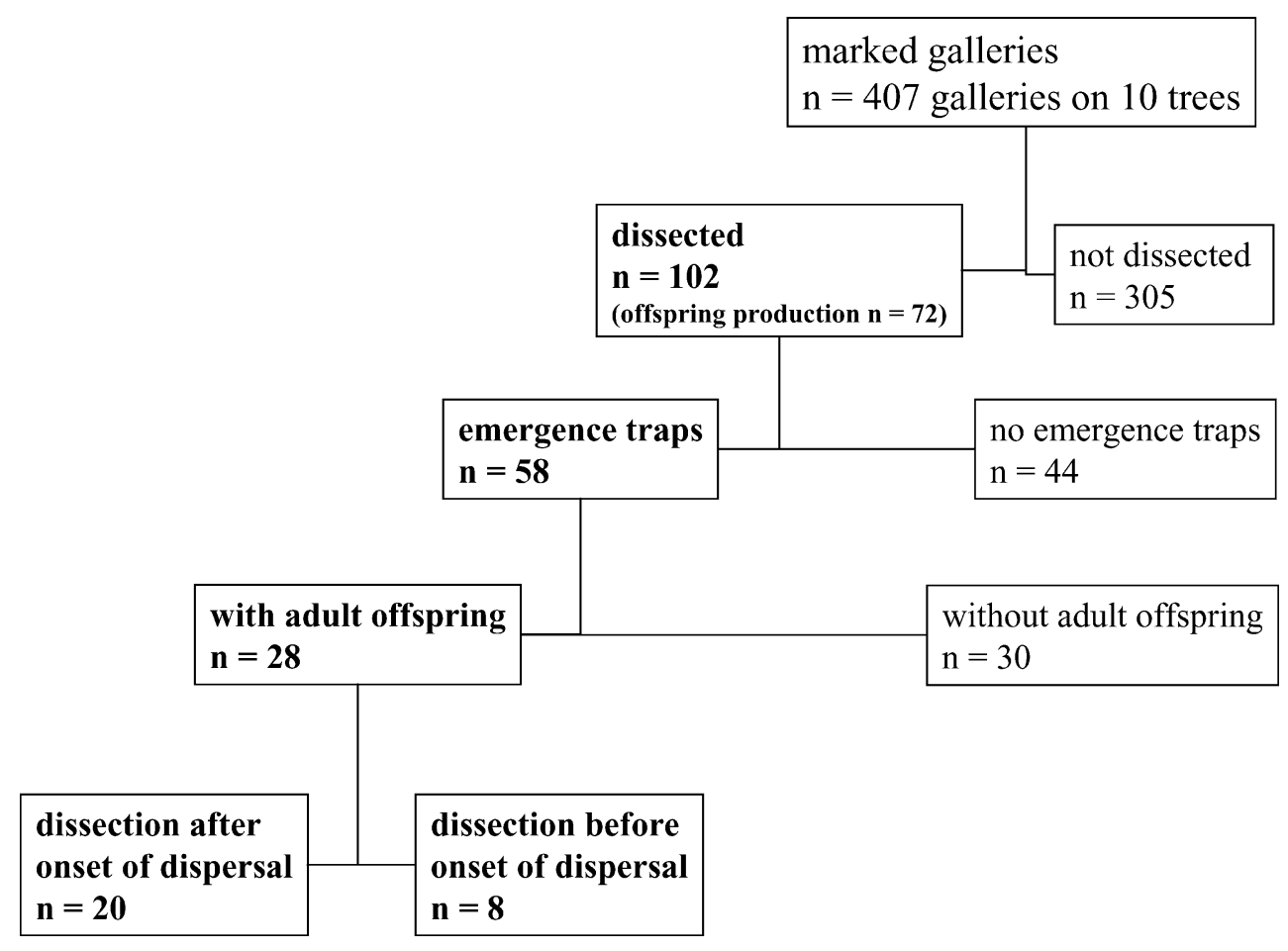


to test for correlations between different stages. To identify important predictors for the occurrence of dispersal, we performed a forward stepwise logistic regression with binomial error distribution. Dispersal rate and female effect on gallery productivity were analysed using multiple linear regressions. The residuals did not deviate from a normal distribution $(p>0.05$, one-sample Kolmogorov-Smirnov test). All analyses were performed with SPSS (release 11.0, CSPSS, 1989-2001).

\section{Results}

\section{Life history}

\section{Dispersal pattern}

Population-wide dispersal activity ranged from April to September. There was a dispersal peak in spring, but flight activity continued until autumn (Fig. 2). More than $40 \%$ of females dispersed before or after the peak dispersal time in June. The occurrence of dispersal throughout the season was confirmed by dispersal data from galleries where dispersal had already started. Neither the onset of dispersal nor the average number of dispersing individuals showed a single peak (Fig. 3). However, during winter (October to March), there was no indication of dispersal from both trap

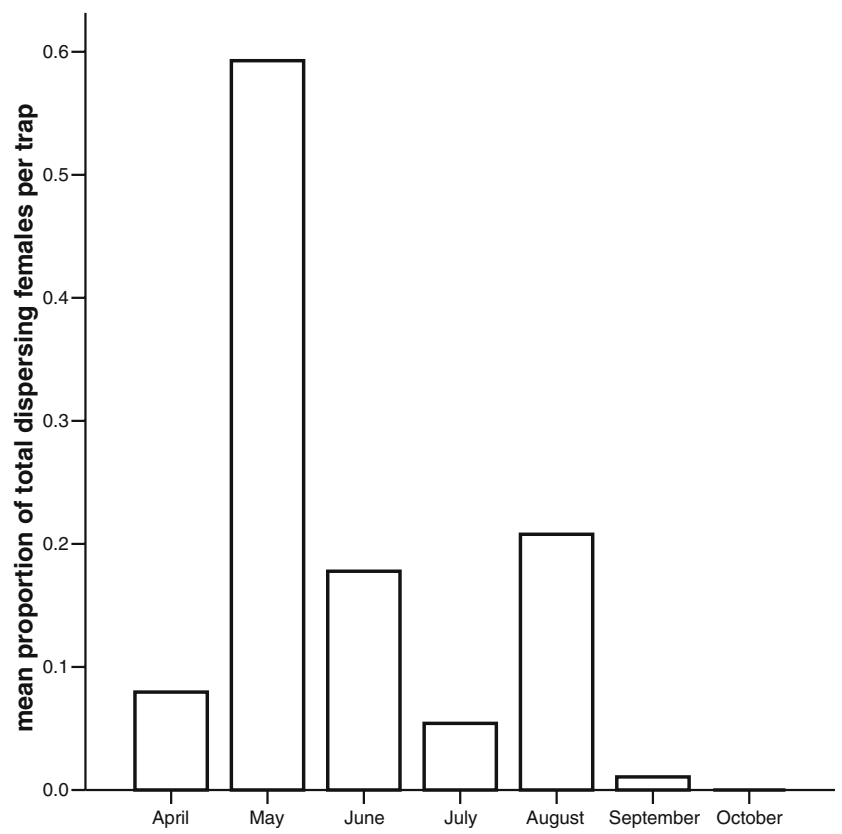

Fig. 2 Population-wide dispersal activity occurs throughout the season. The graph shows the proportion of females dispersing in a particular month relative to the total number of dispersers over the year. Bars represent means per trap. Data of both study years are pooled ( $n=11$ traps) and gallery data. These data show that dispersal (and thus colony foundation) is possible at any time from late spring to early fall (April through September).

Dispersal success can be estimated using the failure rate of marked initiated galleries, which can be regarded as attempts at colony foundation. Of 408 galleries marked on ten different trees, $322(79 \%)$ had either failed by the end of the study period (e.g. death of foundress, no ambrosia growth or contamination with other fungi) or had produced no offspring by the time of dissection. This measure of dispersal success is conservative, as we do not have data on mortality during dispersal flight itself or on potential gallery failure after the end of the study period or the time of dissection. Thus, it is likely that less than about $10 \%$ of all dispersing females succeed in gallery establishment and the raising of their own broods.

In eight galleries, the presence of dead adult female offspring that had not dispersed (on average, 12 individuals) could be determined at the time of dissection (i.e. we could exclude the possibility that they had died because of damage during gallery dissection). In four of these eight galleries, dispersal had been monitored beforehand. In these, $25 \pm 17 \%$ (mean $\pm \mathrm{SD})$ of all females produced (including those that had already dispersed) were dead. Two of these galleries were empty, except for the dead females, indicating completion of dispersal and the end of gallery life. They contained 13 and 12 dead females and had produced 38 and 104 dispersing females, respectively, during 1 year of monitoring their dispersal patterns. These numbers suggest that relatively large proportions of females may never disperse.

\section{Opportunities for cooperative brood care}

We examined whether females would have opportunities to gain inclusive fitness benefits by delayed dispersal. A precondition for cooperative brood care is that adult females and immature siblings must co-occur within galleries. For an analysis of co-occurrence, all data on gallery composition from October until April were pooled because low winter temperatures lessen the likelihood of changes in gallery composition. Total brood size was lowest in June, implying that most galleries were founded during this month. It gradually increased until August, when mean total brood size of dissected galleries was highest, before decreasing steadily until the following June (Fig. 4). This decrease was not due to female mortality, as dead individuals were included in the respective estimates. Instead, it may have been caused by the progressive dispersal of old female offspring. Our results show that all age stages, including adult females and dependent offspring such as eggs, larvae and pupae, were simultaneously present within galleries throughout the year. 

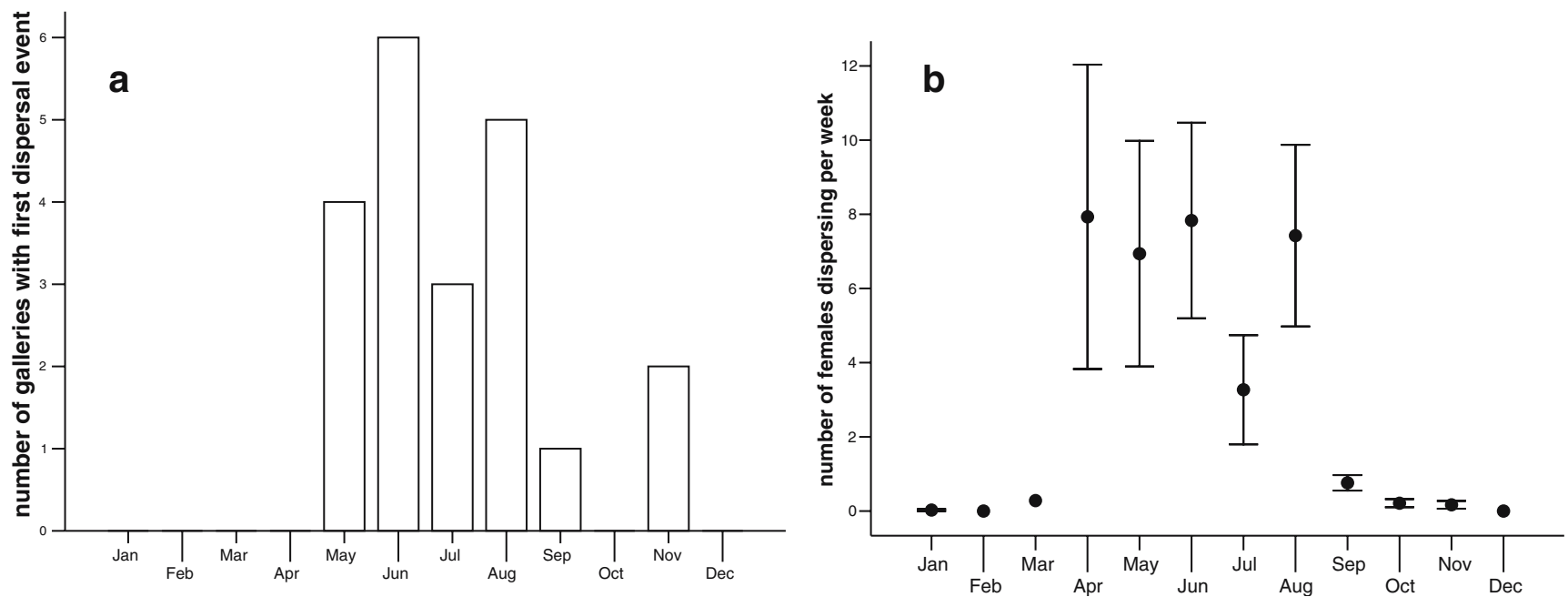

Fig. 3 Dispersal from galleries occurs throughout the season ( $n=20$ galleries). Data of both years are pooled. a Number of galleries with first dispersal event per month. b Mean $( \pm \mathrm{SEM})$ weekly number of dispersing individuals per gallery per month (after the onset of dispersal)

Gallery composition with and without adult female offspring is shown in the Appendix. After maturation of the first female offspring, immature offspring of different ages - and thus opportunities for providing brood carewere present in $37-69 \%$ of the galleries (number depending on age cohort). The mean number of immature individuals was higher with adult females present than without (see Appendix).

These results demonstrate that adult female offspring have the opportunity either to disperse throughout the season or to raise siblings within their natal gallery. This raises the question as to which factors may lead to their eventual dispersal.

\section{Determinants of dispersal propensity}

Females should time their dispersal depending on the relative benefits of staying in their natal gallery and

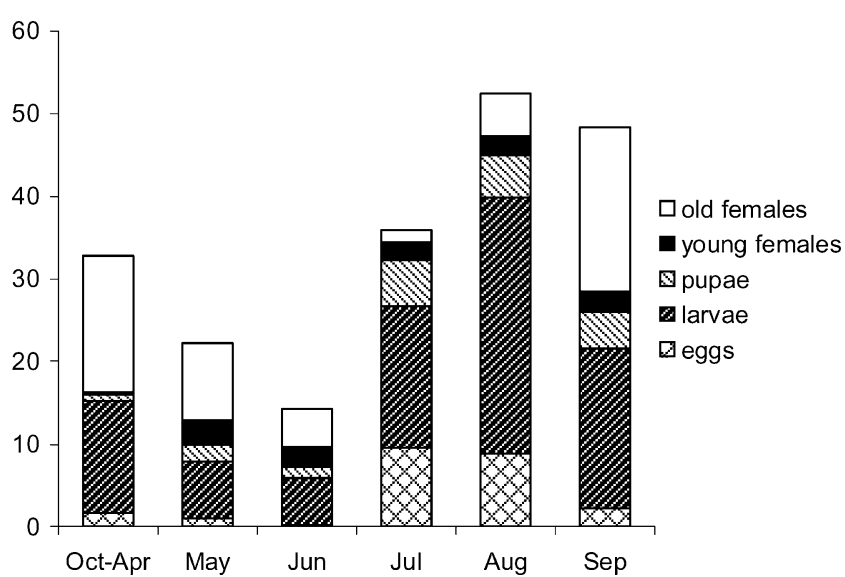

Fig. 4 Throughout the season, adult females as well as dependent offspring are present in galleries. Average number of individuals per age cohort and month are shown ( $n=102$ galleries) dispersing to found their own gallery. We searched for predictors of female dispersal at galleries where adult female offspring were present. We performed a forward conditional logistic regression using the number of individuals from egg to pupal stages at the time of gallery dissection as explanatory variables. The two variables included in the final model were number of eggs ( $\Delta$ deviance $=6.05$, $p=0.014)$ and number of the youngest larval stage L1 ( $\Delta$ deviance $=4.48, p=0.034$ ). These are the youngest age cohorts, which are likely to be influenced by egg production more than by received brood care. The likelihood of dispersal to have already started increased with decreasing numbers of eggs and L1, i.e. with decreasing brood production (Fig. 5).

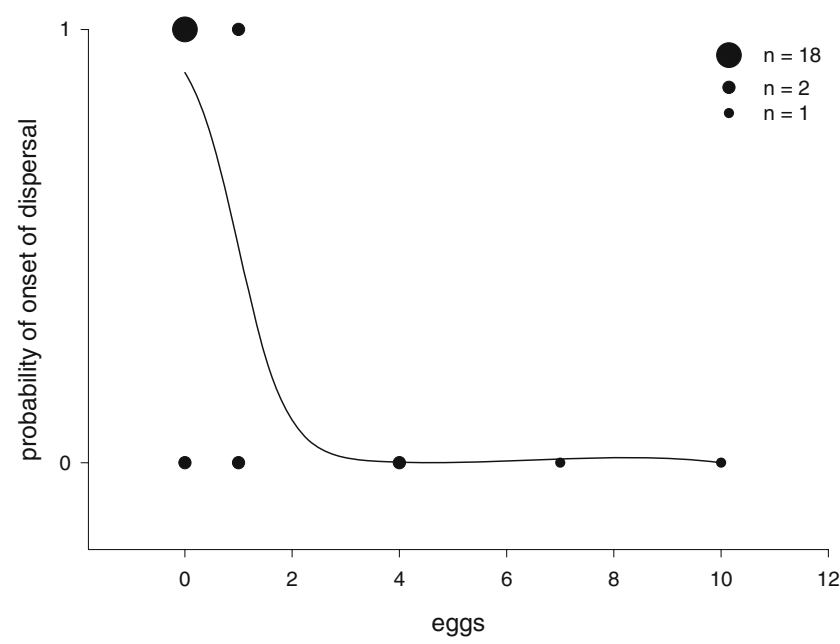

Fig. 5 More galleries have initiated dispersal after brood production has ceased. The graph shows the relationship between the probability of onset of dispersal at a gallery and the number of eggs inside the gallery (logistic regression line: $\ln (\mathrm{p})=2.201-2.212 \times$ eggs, $n=28$ galleries). In the final model, the youngest larval stage was also included 


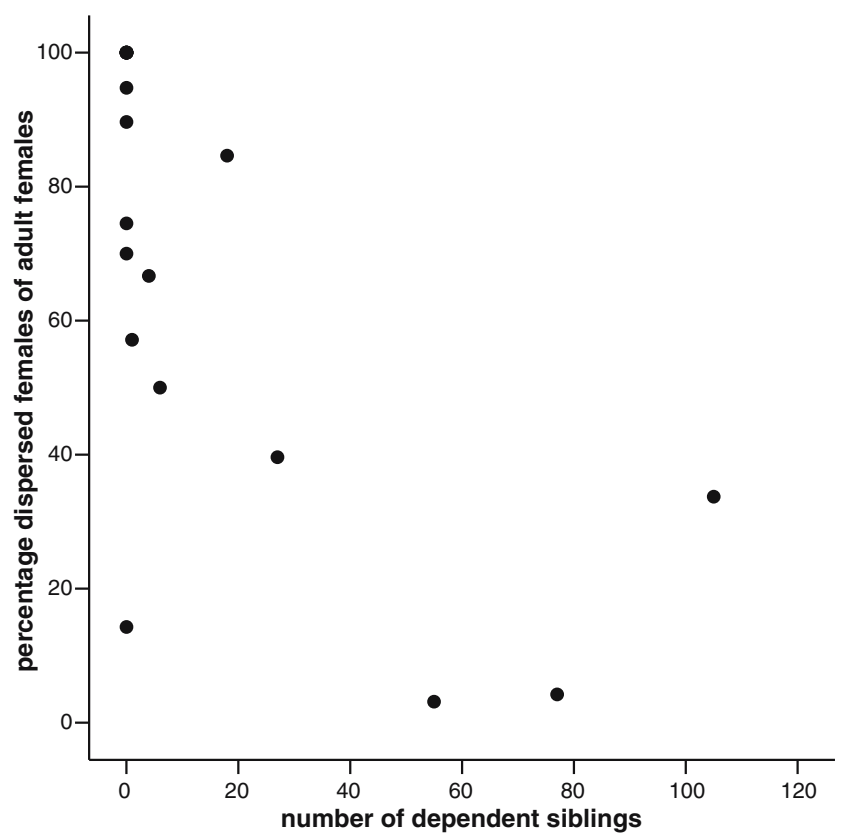

Fig. 6 More females disperse when fewer dependent siblings are present in the gallery. The relationship is shown between the number of dependent siblings and the proportion of females that have already dispersed at the time of dissection. Only old females, which were sclerotized and thus able to disperse, were included in the analysis (regression equation: dispersed females $=1.629+0.67 \times$ old females $-0.523 \times$ dependent siblings, $n=20$ galleries; some points in the graph overlap, giving the impression of a smaller sample size)

Once dispersal had started, the number of dependent brood (larvae and pupae) in the gallery was significantly related to dispersal rate: there was a negative relationship between the sum of all dispersed females and the number of dependent siblings present at the time of dissection. The effect of colony size was accounted for by including the total number of old (sclerotized) females produced (still present plus dispersed) as a covariant (multiple regression; dependent siblings: $t=-4.157, p=0.001$; total number of old females: $t=6.747, p<0.001$; Fig. 6 ).

Potential roles of adult females in the colony

One potential benefit of delayed dispersal is participation in reproduction within the natal gallery. However, the number of eggs was negatively related to the number of old females present in a gallery (Spearman's $r_{\mathrm{s}}=-0.24, p=0.042$ ) and, although not significantly, to the total number of females present (Spearman's $r_{\mathrm{s}}=-0.211, p=0.075$; Fig. 7). These data are consistent with the hypothesis that usually only a single reproductive female (most likely the foundress) exists per colony, suggesting a maximal reproductive skew within galleries. Only one gallery (S2.29) contained a very high number of eggs (108), which may hint at an occurrence of egg laying by multiple females.

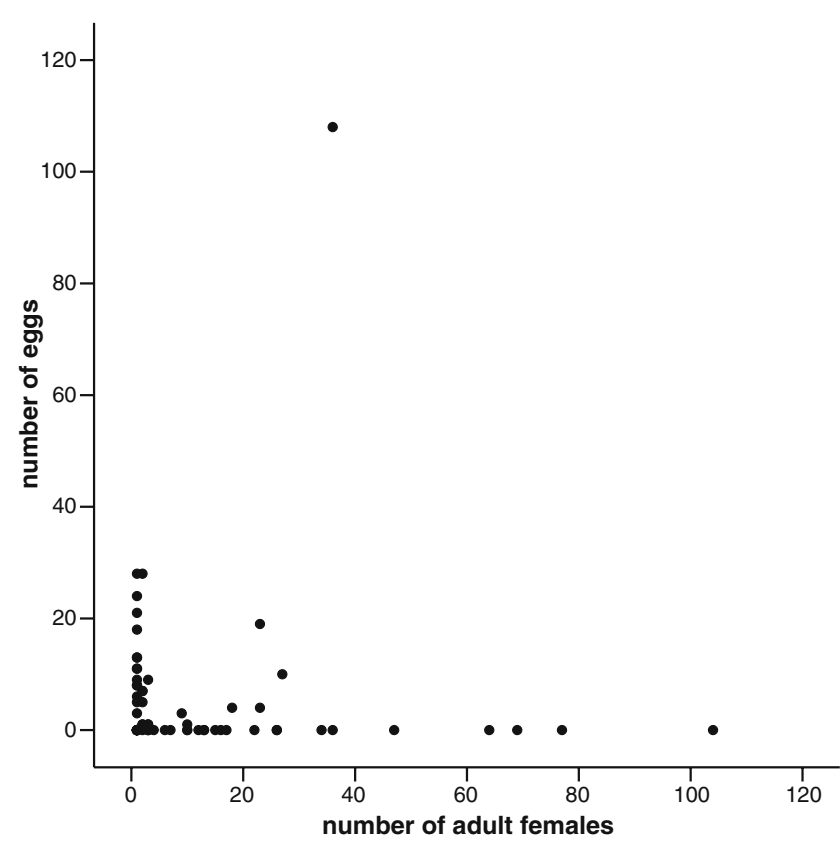

Fig. 7 Egg number does not increase with the number of females. The abscissa represents the number of adult females that were inside the gallery at the time of dissection ( $n=72$ galleries). The outlying data point represents gallery S2.29 with an unusually high number of eggs (see text)

Another potential benefit of delayed dispersal is to help in raising relatives. If we are interested in the potential effect of adult females on the survival of siblings, we need to control for differences in the number of offspring produced in a gallery. Therefore, we included the number of individuals contained in the age class preceding the target age class (i.e. the dependent variable) as a covariate in the analysis. Only galleries where the age cohort under examination was

Table 1 Results of a multiple regression analysis testing for a relationship between the number of females present in a colony and the number of dependent siblings (larvae and pupae)

\begin{tabular}{llclll}
\hline $\begin{array}{l}\text { Dependent } \\
\text { variable }\end{array}$ & $\begin{array}{l}\text { Predictors } \\
\text { and } \\
\text { covariates }\end{array}$ & $\begin{array}{l}n \\
\text { galleries }\end{array}$ & $\begin{array}{l}B \\
\text { (unstandardized } \\
\text { coefficient) }\end{array}$ & & \\
\hline Larvae & & 49 & & & \\
& Eggs & & 0.668 & 3.194 & 0.003 \\
& Females & & 0.321 & 2.092 & 0.042 \\
Pupae & & 31 & & & \\
& Larvae & & 0.192 & 3.732 & 0.001 \\
& Females & & 0.183 & 3.035 & 0.005 \\
& & & & & \\
\hline
\end{tabular}

The age cohorts preceding the respective dependent stage (number of eggs for the larval stage and number of larvae for the pupal stage) were included as covariates. 

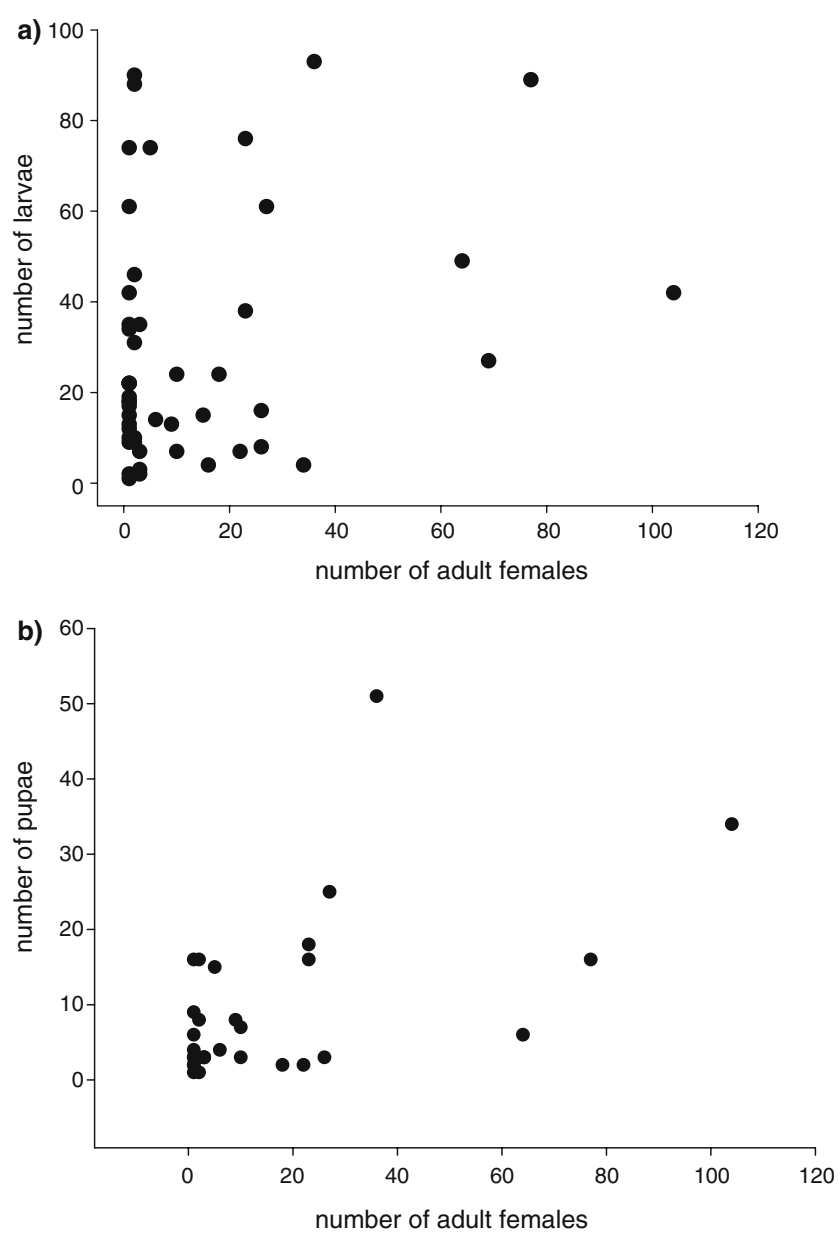

Fig. 8 The number of dependent siblings is greater in galleries with a large number of adult females, which suggests a helper effect. The relationship is shown between the number of adult females present in a gallery and $\mathbf{a}$ the number of larvae ( $n=49$ galleries) and $\mathbf{b}$ the number of pupae $(n=31$

galleries)

present were included. Both the total number of larvae and the number of pupae were significantly positively related to the number of females present in the gallery, independently of the number of individuals in the respective preceding age class (multiple regression; Table 1, Fig. 8a,b). This suggests that the presence of adult female offspring enhances survival of dependent siblings.

In addition to this positive effect of adult females on sibling survival, differences in the intrinsic quality of galleries might influence the number of individuals in a gallery. If such significant habitat differences exist, the size of colonies (i.e. total number of individuals in a gallery) should correlate with colonization density. However, there was no correlation between colony size and the distance to the nearest gallery (Pearson's $r=0.1, p=0.53, n$ $=39$ ).

\section{Discussion}

Our results show that adult females of $X$. saxeseni delay dispersal from their natal galleries after reaching adulthood, which is one of three key decisions an individual has to take in a social trajectory towards cooperative breeding. Furthermore, the data suggest that females do not reproduce in their natal gallery and that their presence enhances gallery productivity, which indicates helping behaviour.

In $X$. saxeseni, dispersal occurs (and hence is possible) at any time during summer and fall. However, throughout the year, a substantial number of adult female offspring remains in their natal gallery. The presence of dead females within galleries indicates that some individuals may entirely refrain from dispersal. Regardless whether the death of these females was caused by disease or other causes, they did not disperse, although they were physically ready to do so. Hosking (1972) also reported that in $10 \%$ of all galleries, dead individuals were present after completion of dispersal, without giving information on the proportion of dead females per gallery.

What is the reason that females delay dispersal after reaching sexual maturity? They might need to feed on ambrosia for some time after emergence to achieve sclerotization (as suggested by McNee et al. 2000 for phloem-feeding bark beetles). However, this would not explain the high proportion of dark (i.e. sclerotized) females in the colony (see Appendix). A further possibility would be that females accumulate energy reserves before dispersal. We have no information about body condition and dispersal success in $X$. saxeseni, but a lab study of a closely related species (Xylosandrus germanus) revealed that females successfully found new galleries without consuming food in their natal gallery after emergence from the pupal stage (Peer and Taborsky 2005). Finally, it is unlikely that adult female offspring usually gain considerable direct fitness benefits through individual reproduction, as there was no positive relationship between the number of females and the number of eggs laid in a gallery. Data on ovarian development of adult female offspring are required to test this assumption, but preliminary results from dissections show that dispersing females usually do not have developed ovaries (Biedermann, personal communication). Only a single, very large colony (with a total brood size of 291) contained a substantially larger number of eggs than all others, indicating an exceptional case where egg laying by female offspring may have occurred. As there are no obvious morphological differences between females (unpublished data), totipotency of females and some flexibility of the breeding system can be assumed.

Most galleries with adult female offspring also contained immature individuals, which may benefit from brood care 
by their adult sisters (see Appendix). Our data suggest that dispersal starts when the production of new offspring ceases (i.e. when eggs and freshly hatched larvae are missing). We have no information about the cause of termination of egg laying, but it is possible that dehydration of the wood leads to deterioration of fungus, which will reduce the survival prospects of newly produced offspring. Fischer (1954) reported dispersal of $X$. saxeseni from logs that were dehydrated due to storage conditions, whereas there was no dispersal when logs were stored on moist ground. A similar situation was described for a drywood termite species: diminished resource availability resulted in helpers changing their tactic and becoming dispersing reproductives (Korb and Lenz 2004). After the onset of dispersal from a gallery had started in $X$. saxeseni, the number of dependent offspring still present in the gallery was negatively related to the number of dispersers. This correlation may suggest that dispersal decisions depend on the amount of help needed in the natal gallery, but it may also be a fortuitous side effect if newly emerged adults remain in the nest for some other reason, and the number of dependent brood declines due to colony aging during the same time period. However, any coincidence between female dispersal and the number of siblings in need of help provides opportunities for positive fitness effects owing to cooperation.

Positive effects of cooperative brood care are suggested by the significant relationship between the number of females in a gallery and the number of larvae and pupae. As there is no correlation with egg number, this suggests that adult female offspring generally do not produce own offspring but instead raise the probability of sibling survival. Colony size was not associated with colonization density, which makes it unlikely that site quality rather than the presence of females was responsible for the described relationship. Provided this interpretation will be confirmed by future studies, $X$. saxesen $i$ can be viewed as a communal (cooperation in brood care) or cooperative breeder (alloparental brood care), following the classification of Crespi and Choe (1997).

There are several potential tasks through which females may contribute to colony success. The ambrosia fungus is cultivated on the surface of the gallery walls (Hosking 1972; Kirkendall et al. 1997). Excavation and enlargement of the brood chamber will increase the space and food resources available for larvae, thus creating benefits for relatives without increasing local competition. Enlargement of the gallery by adult offspring has been suggested for a number of xyleborine species (Saunders et al. 1967; Gagne and Kearby 1979; Schneider 1987; Merk1 and Tusnadi 1992) including X. saxeseni (Hopkins 1898; Fischer 1954). In addition, ambrosia growth has to be controlled so that the fungus will not overgrow the whole chamber, and cropping by females or special secretions may play an important role (Hadorn 1933; Batra 1966). Finally, eggs, larvae and pupae have to be cleaned, and faeces and other debris must be removed from the gallery (Kingsolver and Norris 1977). Help by adult female offspring may allow the foundress to invest more energy into egg laying.

The contribution of female helpers needs to be quantified by behavioural observations in future studies of $X$. saxeseni. Direct observations in the field are impossible due to the concealed lifestyle of Xyleborini, but observations may be possible through cultivation in artificial medium in the lab (e.g. Roeper et al. 1980; Bischoff 2004). We have been able to breed and observe a closely related species in artificial medium (X. germanus; Peer and Taborsky 2004), where adult female offspring were found to spend a considerable proportion of time on cleaning behaviour before dispersal (Bischoff 2004). Unfortunately, lab cultures of $X$. saxesen $i$ were much less successful. Extensive attempts to optimize the culture medium have been minimally successful, and first qualitative observations confirmed that adult female offspring do indeed tend fungi in their natal gallery (P. Biedermann, personal communication).

Which factors may be important in the evolution of delayed dispersal and cooperative brood care in $X$. saxeseni and other Xyleborini? If population regulation is locally plastic, i.e. more altruistic patches consisting of cooperative individuals are able to sustain higher densities, limited dispersal and inbreeding can favour altruism (e.g. Taylor 1992a,b; Mitteldorf and Wilson 2000). This situation applies to the Xyleborini, as an enlargement of brood chambers by adult offspring would permit larger brood sizes. Inbreeding strongly affects population structure by decreasing within-family variance and selection, whereas increasing between-family variance (Wade 1980). Therefore, inbreeding is predicted to enhance the evolution of social behaviour through group selection (see also Wilson 2001). In line with this prediction, intense inbreeding has been found at the origin of eusociality in gall-inducing thrips (Chapman et al. 2000), and eusocial aphid colonies constitute a single clone because they are derived from a single foundress (e.g. Aoki 1977). Similarly, eusocial naked mole rats, social spider mites and social spiders are characterized by high levels of inbreeding (Reeve et al. 1990; Smith and Hagen 1996; Saito 1997). In Xyleborini, levels of inbreeding are likely to be extremely high owing to their mating system (e.g. inbreeding coefficients of about 0.9 in $X$. germanus, own unpublished genetic data), probably comparable to those of eusocial thrips. Because of these high levels of inbreeding, which reduce relatedness asymmetries caused by haplodiploidy and increase overall relatedness, there 
may be little or no conflict over reproduction within xyleborine colonies.

The costs and benefits associated with the decisions of dispersal, individual reproduction and helping are largely determined by ecological factors. In $X$. saxeseni, constraints on individual reproduction are probably severe because a risky dispersal flight is required, and finding a suitable host location is uncertain. In addition, our data suggest that already-initiated galleries have a failure rate of $80 \%$. Hosking (1972) also reported very high failure rates $(40-60 \%)$. It remains to be determined whether this rate differs between xyleborine species. $X$. saxeseni appears to be particularly selective regarding the required condition of host trees: it preferentially colonizes very recently dead trees that are often still standing (personal observation). This apparent selectivity may decrease the chances of finding a suitable host. Furthermore, the rather deep penetration of the entrance tunnel into the wood compared to other temperate Xyleborine species may require large initial investment for gallery construction and thus increase the cost of dispersal.

In ambrosia beetles, potential benefits of helping in the natal gallery are associated with the cultivation of fungus. This is a universal habit of all xyleborine species, and cooperative breeding may be a widespread phenomenon among them. A critical ecological factor determining the social system is probably gallery longevity: if brood chambers persist long enough to allow overlap between immature and mature offspring, helping in the natal gallery can be a profitable alternative to dispersing (Kirkendall et al. 1997). The galls of eusocial, gall-forming thrips are longer-lived than those of nonsocial species (Crespi and Mound 1997). The only species of ambrosia beetle that has been suggested to be eusocial, Austroplatypus incompertus, is special among these beetles, as it attacks live trees, and a colony may persist more than 30 years within a living tree (Kent and Simpson 1992). X. saxeseni has been reported to sometimes attack live trees (Hopkins 1898; Fischer 1954), and its entrance tunnels can lead several centimetres into the inner wood of a tree. This is likely to enhance the temporal stability of the brood chamber for two reasons: dehydration proceeds from the periphery towards the centre of a log, and thus, fungus growth in deeply excavated burrows may be maintained for a longer period of time. Furthermore, the insulating effects of the wood will protect the brood chamber from very low temperatures during winter, so that overwintering is also possible for immature offspring (Fischer 1954). A prolonged phase of fungus growth and brood production together with cooperative brood care by adult offspring might also explain the unusually large brood sizes of $X$. saxeseni compared to those of other European Xyleborini (Hosking 1972).

The rapid radiation of the Xyleborini together with their ecology and high sociality potential makes this tribe a promising candidate for future studies of the evolution of cooperative breeding and eusociality. The recent clarification of the phylogeny of these beetles and the coevolution with their fungal symbionts (Normark et al. 1999; Jordal et al. 2000; Farrell et al. 2001) provide an ideal background for comparative studies of the relative influence of various ecological factors on the evolution of cooperative breeding.

Acknowledgment We thank Ralph Bergmueller, Laurent Keller, Lawrence Kirkendall and anonymous referees for comments on previous drafts of the manuscript, and Dik Heg and Barbara Tschirren for statistical advice. The investigations comply with the current laws of Switzerland.

\section{Appendix}

Table 2 Gallery composition in the presence and absence of adult female offspring

\begin{tabular}{|c|c|c|c|c|c|c|c|c|c|}
\hline $\begin{array}{l}\text { Presence of adult } \\
\text { female offspring }\end{array}$ & & Eggs & $\mathrm{L} 1$ & L2 & L3 & Total larvae & Female pupae & Young females & Old females \\
\hline \multirow[t]{2}{*}{ No } & Mean & 5.7 & 2.5 & 3.7 & 8.2 & 14.4 & 1.5 & & \\
\hline & Percent galleries with & 45.2 & 38.7 & 54.8 & 51.6 & 61.3 & 29.0 & & \\
\hline \multirow[t]{2}{*}{ Yes } & Mean & 5.1 & 1.9 & 3.3 & 17.5 & 22.7 & 5.6 & 3.9 & 14.78 \\
\hline & Percent galleries with & 39.0 & 43.9 & 51.2 & 68.3 & 70.7 & 51.2 & 56.1 & 85.4 \\
\hline
\end{tabular}

Gallery composition in the presence $(n=41)$ and absence $(n=31)$ of adult female offspring (i.e. excluding the colony foundress). Only galleries that had produced any offspring at the time of dissection are included. For each age cohort, the mean number of individuals per colony is given together with the percentage of galleries in which this age cohort was found.

$\mathrm{L} 1-\mathrm{L} 3=$ the three larval stages. 


\section{References}

Aanen DK, Eggleton P, Rouland-Lefévre C, Guldberg-Frøslev T, Boomsma JJ, Rosendahl S (2002) The evolution of fungusgrowing termites and their mutualistic fungal symbionts. Proc Natl Acad Sci USA 99:14887-14892

Alexander RD, Noonan KM, Crespi BJ (1991) The evolution of eusociality. In: Sherman PW, Jarvis JUM, Alexander RD (eds) The biology of the naked mole rat. Princeton University Press, Princeton, pp 1-44

Aoki S (1977) Colophina clematis (Homoptera, Pemphigidae), an aphid species with soldiers. Kontyu 45:276-282

Batra LR (1966) Ambrosia fungi: extent of specificity to ambrosia beetles. Science 153:193-195

Bignell D, Eggleton P (2000) Termites: evolution, sociality, symbioses, ecology. Kluwer, Dordrecht, The Netherlands

Bischoff LL (2004) The social structure of the haplodiploid bark beetle, Xylosandrus germanus. MSc Thesis, University of Berne, Switzerland

Chapman TW, Crespi BJ, Kranz BD, Schwarz MP (2000) High relatedness and inbreeding at the origin of eusociality in gallinducing thrips. Proc Natl Acad Sci USA 97:1648-1650

Crespi BJ (1992) Eusociality in Australian gall thrips. Nature 359:724-726

Crespi B, Choe JC (1997) Introduction. In: Choe JC, Crespi B (eds) The evolution of social behavior in insects and arachnids. Cambridge University Press, Cambridge, pp 1-7

Crespi BJ, Mound LA (1997) Ecology and evolution of social behaviour among Australian gall thrips and their allies. In: Choe JC, Crespi BJ (eds) The evolution of social behavior in insects and arachnids. Cambridge University Press, Cambridge, pp 166-180

Duffy JE (1996) Eusociality in a coral-reef shrimp. Nature 381:512-514

Eichhoff W (1881) Die Europäischen Borkenkäfer. Julius Springer, Berlin

Emlen ST (1994) Benefits, constraints and the evolution of the family. Trends Ecol Evol 9:282-285

Farrell BD, Sequeira AS, O'Meara BC, Normark BB, Chung JH, Jordal BH (2001) The evolution of agriculture in beetles (Curculionidae: Scolytinae and Platypodinae). Evolution 55: 2011-2027

Fischer M (1954) Untersuchungen über den kleinen Holzbohrer (Xyleborus saxeseni). Pflanzenschutzberichte 12:137-180

Gadagkar R (1990) Evolution of eusociality: the advantage of assured fitness returns. Phil Trans R Soc Lond B 329:17-25

Gagne JA, Kearby WH (1979) Life history, development and insecthost relationships of Xyleborus celsus (Coleoptera: Scolytidae) in Missouri. Can Entomol 111:295-304

Hadorn C (1933) Recherche sur la morphologie, les stades évolutifs et l'hivernage du bostryche liseré (Xyloterus lineatus Oliv.). Z Schweiz Forstvereins (Suppl) 11:1-20

Helms Cahan S, Sundström L, Liebig J, Griffin A (2002) Social trajectories and the evolution of social behaviour. Oikos 96:206-216

Hopkins AD (1898) On the history and habits of the "wood engraver" ambrosia beetle-Xyleborus xylographus (Say), Xyleborus saxeseni (Ratz.) - with brief description of different stages. Can Entomol 30:21-29

Hosking GB (1972) Xyleborus saxeseni, its life-history and flight behaviour in New Zealand. N Z J For Sci 3:37-53

Jordal BH, Normark BB, Farrell BD (2000) Evolutionary radiation of an inbreeding haplodiploid beetle lineage (Curculionidae, Scolytinae). Biol J Linn Soc 71:483-499

Kent DS, Simpson JA (1992) Eusociality in the beetle Austroplatypus incompertus (Coleoptera: Platypodidae). Naturwissenschaften 79:86-87

Kingsolver JG, Norris DM (1977) The interaction of Xyleborus ferrugineus (Fabr.) (Coleoptera: Scolytidae) behavior and initial reproduction in relation to its symbiotic fungi. Ann Entomol Soc Am 70:1-4
Kirkendall LR (1993) Ecology and evolution of biased sex ratios in bark and ambrosia beetles. In: Wrensch DL, Ebbert MA (eds) Evolution and diversity of sex ratio in insects and mites. Chapman \& Hall, New York, pp 235-345

Kirkendall LR, Kent DS, Raffa KF (1997) Interactions among males, females and offspring in bark and ambrosia beetles: the significance of living in tunnels for the evolution of social behavior. In: Choe JC, Crespi BJ (eds) The evolution of social behavior in insects and arachnids. Cambridge University Press, Cambridge, pp 181-215

Koenig WD, Pitelka FA, Carmen WJ, Mumme RL (1992) The evolution of delayed dispersal in cooperative breeders. Q Rev Biol 67:111-150

Korb J, Lenz M (2004) Reproductive decision-making in the termite, Cryptotermes secundus (Kalotermitidae), under variable food conditions. Behav Ecol 15:390-395

McNee WR, Wood DL, Storer AJ (2000) Pre-emergence feeding in bark beetles (Coleoptera : Scolytidae). Environ Entomol 29:495-501

Merkl O, Tusnadi CsK (1992) First introduction of Xyleborus affinis (Coleoptera: Scolytidae), a pest of Dracaena fragrans 'Massangeana', to Hungary. Folia Entomol Hung 52:67-72

Mitteldorf J, Wilson DS (2000) Population viscosity and the evolution of altruism. J Theor Biol 204:481-496

Normark BB, Jordal BH, Farrell BD (1999) Origin of a haplodiploid beetle lineage. Proc R Soc Lond B 266:2253-2259

Peer K, Taborsky M (2004) Female ambrosia beetles adjust their offspring sex ratio according to outbreeding opportunities for their sons. J Evol Biol 17:257-264

Peer K, Taborsky M (2005) Outbreeding depression, but no inbreeding depression in haplodiploid ambrosia beetles with regular sibling mating. Evolution 59:317-323

Reeve HK, Westneat DF, Noon WA, Sherman PW, Aquadro CF (1990) DNA 'fingerprinting' reveals high levels of inbreeding in colonies of the eusocial naked mole rat. Proc Natl Acad Sci USA 87:2496-2500

Roeper R, Treeful LM, O’Brien KM, Foote RA, Bunce MA (1980) Life history of the ambrosia beetle Xyleborus affinis (Coleoptera: Scolytidae) from in vitro culture. Great Lakes Entomol 13:141-144

Roisin Y (1999) Philopatric reproduction, a prime mover in the evolution of eusociality? Insectes Soc 46:297-305

Saito Y (1997) Sociality and kin selection in Acari. In: Choe JC, Crespi BJ (eds) The evolution of social behavior in insects and arachnids. Cambridge University Press, Cambridge, pp 443-457

Saunders JL, Norris DM, Knoke JK (1967) Insect-host tissue interrelations between Xyleborus ferrugineus (Coleoptera: Scolytidae) and Theobroma cacao in Costa Rica. Ann Entomol Soc Am 60:419-423

Schneider I (1987) Verbreitung, Pilzübertragung und Brutsystem des Ambrosiakäfers Xyleborus affinis im Vergleich mit X. mascarensis (Coleoptera: Scolytidae). Entomol Gen 12:267-275

Schwenke W (1974) Die Forstschädlinge Europas. Paul Parey, Hamburg

Sherman PW, Lacey EA, Reeve HK, Keller L (1995) The eusociality continuum. Behav Ecol 6:102-108

Smith DR, Hagen RH (1996) Population structure and interdemic selection in the cooperative spider Anelosimus eximius. J Evol Biol 9:589-608

Taylor PD (1992a) Altruism in viscous populations - an inclusive fitness model. Evol Ecol 6:352-356

Taylor PD (1992b) Inclusive fitness in a homogeneous environment. Proc R Soc Lond B 249:299-302

Thorne BL (1997) Evolution of eusociality in termites. Ann Rev Ecolog Syst 28:27-54

Wade MJ (1980) An experimental study of kin selection. Evolution 34:844-855

West SA, Pen I, Griffin AS (2002) Conflict and cooperation - Cooperation and competition between relatives. Science 296:72-75

Wilson DS (2001) Cooperation and altruism. In: Fox CW, Roff DA, Fairbairn DJ (eds) Evolutionary ecology: concepts and case studies. Oxford University Press, Oxford, pp 222-231 